\title{
Epididymo-Orchitis: A Rare Manifestation of MIS-C
}

\author{
K. C. Sudeep ${ }^{1} \cdot$ R. Muthuvel ${ }^{1} \cdot$ Nazakat Hussain $^{2} \cdot$ Puspraj Awasthi $^{1} \cdot$ Suresh Kumar Angurana ${ }^{1} \cdot$ Arun Bansal $^{1}(\mathbb{C}$
}

Received: 23 August 2021 / Accepted: 12 October 2021 / Published online: 3 November 2021

(c) Dr. K C Chaudhuri Foundation 2021

To the Editor: Multisystem inflammatory syndrome in children (MIS-C) is a postinfectious immunological and hyperinflammatory manifestation of SARS-CoV-2 infection [1]. Genitourinary system involvement in MIS-C is rare.

A 9-y-old male presented with fever, and scrotal pain and swelling for $5 \mathrm{~d}$. There was no history of trauma, urinary symptoms, parotid swelling or pain, coronavirus disease 2019 (COVID-19), or contact with the COVID-19 case. He had fever, shock, respiratory distress, generalized erythematous blanchable rash, hemorrhagic nonpurulent conjunctivitis, and tender erythematous scrotal swelling. He was started on bilevel positive airway pressure (BiPAP) ventilation, vasoactive drugs, and antibiotics. Investigations revealed neutrophilic leucocytosis, lymphopenia, and elevated inflammatory markers. Echocardiography showed a left ventricular ejection fraction of $35 \%$ and normal coronary arteries. Nasopharyngeal swab for SARS-CoV-2 RTPCR was negative, but SARS-CoV-2 serology was positive. Ultrasonography of the scrotum showed increased testicular volume with inhomogeneous pattern and increased flow and inflammation of the epididymis on color Doppler suggestive of epididymo-orchitis. There was no evidence of testicular or appendages torsion. Blood and urine cultures were sterile. Antimumps antibody was negative. The diagnosis of MIS-C with myocardial dysfunction and epididymo-orchitis was considered, and intravenous immunoglobulin, methylprednisolone $(10 \mathrm{mg} / \mathrm{kg} / \mathrm{d}$ for $3 \mathrm{~d})$, and aspirin were given.

Clinical symptoms and inflammatory markers improved by day 3 . He was discharged after $8 \mathrm{~d}$ on prednisolone $(2 \mathrm{mg} /$ $\mathrm{kg} / \mathrm{d}$ for 2-3 wk) and aspirin (4-6 wk). At 1 mo of follow-up, he was asymptomatic.

Arun Bansal

drarunbansal@gmail.com

1 Division of Pediatric Critical Care, Department of Pediatrics, Advanced Pediatrics Center, Postgraduate Institute of Medical Education and Research (PGIMER), Chandigarh 160012, India

2 Resident, Department of Pediatrics, Advanced Pediatrics Center, Postgraduate Institute of Medical Education and Research (PGIMER), Chandigarh, India
Genitourinary manifestations have been noted in $10 \%-20 \%$ of cases of COVID-19, but such manifestations are rare in MIS-C. Genitourinary manifestations in MIS-C are postulated due to cytokine storm and hyperinflammation [2]. Gagliardi et al. reported epididymo-orchitis in a 14-yold boy with COVID-19 [3]. Gümüşer et al. reported acute epididymitis with MIS-C in an 8-y-old boy [4]. Management of epidymo-orchitis in MIS-C is with antibiotics, steroids, and IVIG.

\section{Declarations}

Conflict of Interest None.

\section{References}

1. Kumar AS, Awasthi P, Thakur A, et al. Intensive care needs and short-term outcome of multisystem inflammatory syndrome in children (MIS-C): experience from North India. J Trop Pediatr. 2021;67:fmab055.

2. Liu K, Chen X, Ren X, Wu Y, Ren S, Qin C. SARS-CoV-2 effects in the genitourinary system and prospects of sex hormone therapy. Asian J Urol. 2021;8:303-14.

3. Gagliardi L, Bertacca C, Centenari C, et al. Orchiepididymitis in a boy with COVID-19. Pediatr Infect Dis J. 2020;39:e200-2.

4. Gümüşer Cinni R, Polat M, Seyrek H, Kaynak Şahap S, Öz FN, Tanır G. Acute epididymitis associated with multisystem inflammatory syndrome in children. J Paediatr Child Health. 2021;57:594-5.

Publisher's Note Springer Nature remains neutral with regard to jurisdictional claims in published maps and institutional affiliations. 